\title{
OPEN Couette flow of viscoelastic dusty fluid in a rotating frame along with the heat transfer
}

\author{
Muhammad Bilal ${ }^{1}$, Salaha Khan ${ }^{1}$, Farhad $\mathrm{Ali}^{2,3 凶}$, Muhammad Arif ${ }^{1}$, Ilyas $\mathrm{Khan}^{4}$ \& \\ Kottakkaran Sooppy Nisar ${ }^{5}$
}

Viscoelastic fluid is an advanced fluid which exhibits both elastic and viscous properties. Whereas rotation of viscoelastic fluid is a very complex phenomenon and has an immense amount of applications in engineering and product making industries. Due to various applications in real life researchers are working to understand the rheology of viscoelastic fluids. Viscoelastic dusty fluids are used in gas cooling systems. In nuclear reactors, dusty fluids are used to lower the temperature of the system. Such fluids are also used in centrifugal separators, which separate solid particles from the liquid state, etc. Therefore, in the present study, viscoelastic dusty fluid is analyzed. More precisely free convective Couette flow under the influence of the transversely applied uniform magnetic field in a rotating frame is considered. The subject fluid is driven by the sine oscillations of the upper plate along with the effect of free convection. Due to rotation, the fluid and dust particles have complex velocities which is the sum of primary velocity and secondary velocity. The flow regime is modeled in terms of partial differential equations. To non-dimensionalize the system of governing equations, dimensionless variables have been derived through Buckingham-Pi theorem. The system of partial differential equations is solved through assumed periodic solutions (Poincare-Light Hill Technique). The expressions for skin friction (shear stresses at $y=0$ ) and Nusselt number (the rate of heat transfer) are also calculated. Moreover, parametric influence on Nusselt number, and velocity profile of the fluid and the dust particles, is discussed. It is observed that increase in rotation parameter $\eta$ causes retardation in the velocity of dust particles and the fluid. This is due to the fact that the increase in $\eta$ enhances the Coriolis forces that are in fact inertial forces, causing a retardation in the velocity of the fluid phase and the dust phase.

Multiphase magnetohydrodynamic (MHD) flows are of great importance due to their extremely useful applications like fluidization, MHD generators, dusty plasma devices (DPDs), use of dust in cooling systems, and nuclear reactors. Multiphase flow can also be observed in nature such as sediment transport in rivers, in which suspended particles are the solid phase whereas water is the liquid phase. Multiphase flow can also be seen in human bodies i.e. blood flow in human bodies, in which plasma is the liquid phase and red blood cells are referred as solid. The most simple case in multiphase flow is the two-phase flow, which could be a combination of liquid-gas flow, solid-gas flow, liquid-liquid flow and liquid-solid flow.

Viscoelastic fluids are those fluids which posses both the characteristics of viscosity and elasticity which are the traits of fluids and solids respectively. Viscoelastic materials are extremely good shock absorber, therefore, they have many applications in industrial and biomedical sciences especially in the blood flow. Blood flow is viscoelastic in nature due to its elastic energy which is stored in the circulatory system and then used to produce heat energy. The remaining energy is utilized in the movement of the body and other functions ${ }^{1}$. Oldroyd ${ }^{2}$ investigated the creeping nature of viscoelastic fluid by modeling the governing equation and studied the influence of non-Newtonian fluid flow by using Oldroyd fluid model. Rajagopal and Bhatnagar ${ }^{3}$ studied the Oldroyd fluid model and found analytical solutions.

\footnotetext{
${ }^{1}$ Department of Mathematics, City University of Science and Information Technology, Peshawar, Khyber Pakhtunkhwa, Pakistan. ${ }^{2}$ Computational Analysis Research Group, Ton Duc Thang University, Ho Chi Minh City, Vietnam. ${ }^{3}$ Faculty of Mathematics and Statistics, Ton Duc Thang University, Ho Chi Minh City, Vietnam. ${ }^{4}$ Department of Mathematics, College of Science Al-Zulfi, Majmaah University, Al-Majmaah 11952, Saudi Arabia. ${ }^{5}$ Department of Mathematics, College of Arts and Science, Prince Sattam Bin Abdulaziz University, Wadi Al-Dawaser 11991, Saudi Arabia. ${ }^{凶}$ email: farhad.ali@tdtu.edu.vn
} 
Micheal and Miller ${ }^{4}$ studied the dusty gas and its flow generated by the motion of an infinite plate. They utilized the Saffman formulation ${ }^{41}$ by considering two types of motion of the parallel plate i.e. simple harmonic motion and abrupt change in the resting position of the plane which moves with the uniform velocity. Soo ${ }^{5}$ initiated and analyzed the basic theory of multiphase flow. Venkatesh and Kumara ${ }^{6}$ also worked on the unsteady flow of conducting dusty fluids flowing between vibrating plates along a wavy wall. They found that the velocity profile for fluid as well as for dusty particles is parabolic in nature, and tends to become zero for a larger value of time but at the center of the channel the velocities become minimum. Later Ghosh and Sana ${ }^{7}$ analyzed the motion of dusty fluids with spherical dust particles under the effect of magnetic field which is transversely applied to the flow. Many other researchers like Saffman ${ }^{8}$, Vimala ${ }^{9}$, Healy ${ }^{10}$, Venkateshappa et al. ${ }^{11}$, Gupta and Gupta $^{12}$, Ghosh and Ghosh ${ }^{13}$, Attia and Abdeen ${ }^{14}$, Ghosh and Debnath ${ }^{15}$, Gireesha et al. ${ }^{16}$, etc., investigated the theoretical modeling and experimental measurement of particles phase velocity in a multiphase dusty fluid. Ali et al. ${ }^{17}$ studied the generalized two phase flow of blood consisting of magnetic particles. They considered fractional model with the effect of isothermal heating. This study was mainly focused to understand the impact of magnetic field and its uses in human blood. In another paper, Ali et al. ${ }^{18}$ investigated the effect of heat transfer on the two phase blood flow with embedded magnetic particles. They used the newly developed definition of Caputo Fabrizio fractional derivative. Recently Ali et al. ${ }^{19}$ studied the fluctuating two-phase flow of viscoelastic dusty fluid between two horizontal inelastic plates. The effect of heat transfer with the influence of magnetic field was taken into account. Unlike the published work in this investigation, they concluded that the flow in boundary layer increases with the greater value of magnetic field. More recently, Ali et al. ${ }^{20}$ analyzed the fluctuating unsteady free convective flow of viscoelastic fluid with embedded dust particles under the impact of MHD. They discussed the variation of velocity of dust phase and the fluid phase in boundary layer and in free stream with the dual behavior of magnetic parameter.

In astrophysics, engineering sciences and biomedical sciences MHD free convective flow is of prime importance. Free convective MHD flows are also used in the fluid engineering science side such as, they are used in MHD generators, for cooling systems, combustion chambers, radiators, adjusting blood flow during surgery, etc. Due to the above mentioned immensely valuable applications researchers analyzed and studied this phenomenon in each and every possible corner such as ${ }^{21-29}$, investigated the MHD free convective flow, which is of key importance in real world and has many industrial applications like many exothermic and endothermic chemical reactions, removal of heat from nuclear reactors, storage of edible goods, etc. Sheikh et al. ${ }^{30}$, studied the generalized form of Casson fluid with chemical reaction and compared the two different fractional operators. Khan et al. ${ }^{31}$, discussed the effect of chemical reaction on MHD free convective flow over a moveable plate submerged in a semi-permeable medium. Sahoo et al. ${ }^{32}$ scrutinized the free convective MHD flow of electrically conducting incompressible viscous fluid past over a semi-permeable plate, with the effect of heat transfer. Chamkha ${ }^{33}$ investigated the unsteady MHD flow of suspension in a conductive fluid flowing in a channel, considering the effect of thermal radiation. Kumar et al. ${ }^{34}$ studied micro polar and viscous fluid in a vertical channel. They considered the flow to be fully developed and also considered free convection. They found an increasing behavior of velocity with increasing Grashof to Reynolds number ratio, viscosity ratio and width ratio while a decreasing behavior was noticed with increasing micro polar fluid material parameter. A hydromagnetic dusty flow of an electrically conducting fluid has been studied by Chamkha ${ }^{35}$. He has evaluated the analytical solutions and found that owing the existence of dust in channel, the flow rate of fluid-phase and dust-phase both decreases. In another paper Chamkha ${ }^{36}$ has studied the time dependent flow of electrically conducting dusty-gas between two parallel plates. He has found that decrease in volume flow rate of fluid-phase, volume flow rate of dusty-phase occurs by loading the dust particles in channel. Similarly Chamkha ${ }^{37}$ has also discussed MHD flow in channel with free convection. He has also found various analytical solutions for the velocity profile and temperature profile for difference special cases. Furthermore, the Nusselt number for both walls has been calculated. He concluded that no reverse flow ocurrs when the channel is symmetric.

Rotational flows are of immense importance due to their applications in science, engineering, and in product making industries. The science behind the rotational flow provides the basis and modeling capabilities of many manufactured goods such as jet-engines, Vacuum pumps, centrifugal pumps, etc. In nature, rotational flows can also be seen such as in whirlpools, tropical cyclones, tornadoes, etc.Since the rotational flow is a very complex phenomenon, researchers are trying to understand the science behind it. Therefore Gireesha et al. ${ }^{38}$ analyzed the flow of the boundary layer of a dusty fluid having constant angular velocity. They also considered the effect of time-dependent pressure gradient and Hall current. In this investigation they discussed the effect of Ekman number, magnetic parameter, Hall current parameter and time on the fluid's velocity as well as on the velocity of dust particle. Manjunatha et al ${ }^{39}$ investigated the series solutions for a rotating dusty fluid having unsteady flow with free convection and radiation effect. They observed the effect of different parameters such as Hall current parameters, rotation parameter, magnetic parameter on velocity profile as well as on the thermal boundary layer. Dey ${ }^{40}$ analyzed the rotating Jeffrey dusty fluid flow over a non-conducting semi-permeable plate, considering volume fraction and Hall current effect. Nazibuddin et al. ${ }^{41}$ studied an incompressible viscous fluid having transient MHD flow past a horizontal accelerated porous plate in a rotating system with Hall current. They analyzed the effects of velocity profile by varying different parameters such as accelerating parameters, magnetic parameters, and rotational parameters. Kanch and Jana ${ }^{42}$ scrutinized the effect of Hall current on a hydromagnetic unsteady flow past a rotating disk. They concluded that for a large time period the steady-state is attained by inertial oscillations. The frequency of the inertial oscillations initially increases, after reaching the maximum value the frequency decreases by increasing the Hall current parameter. Rajagopal ${ }^{43}$ analyzed the viscoelastic fluid flowing between rotating disks.

Keeping in view the above cited work, up to the best of our knowledge no work has been done on free convective MHD Couette flow of viscoelastic dusty fluid in a rotating frame. As, evident from the aforesaid salient applications of rotatory viscoelastic dusty fluid it is very important to fully understand the rheology of such 


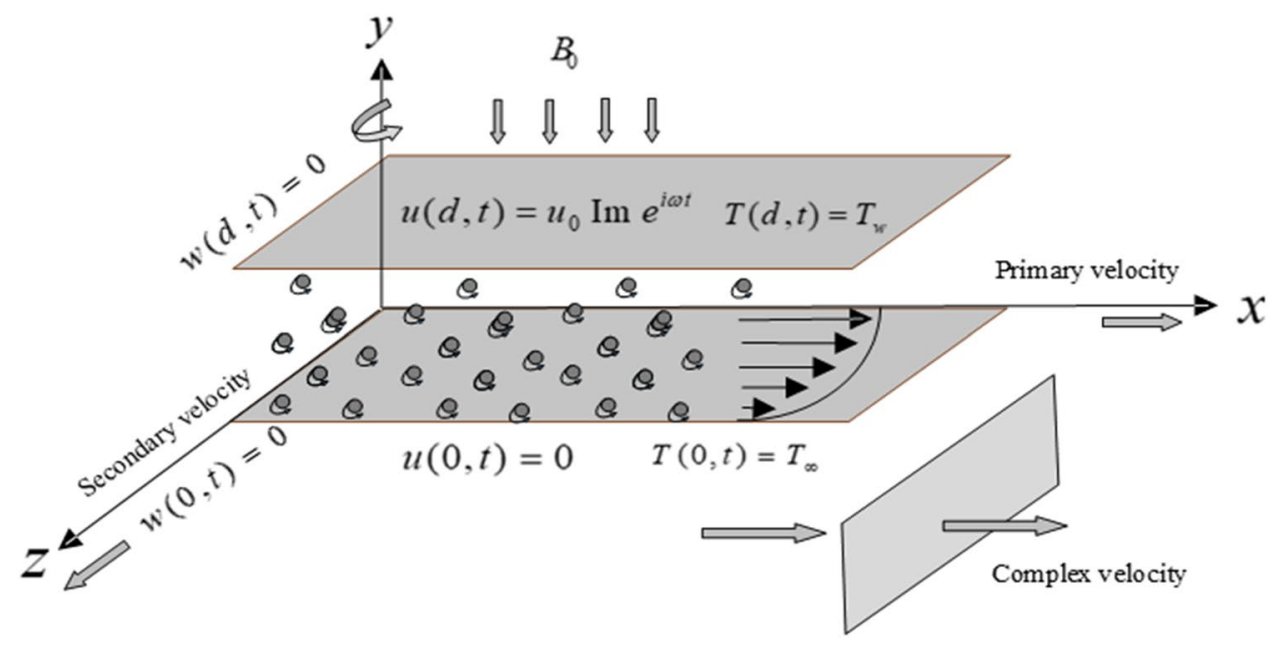

Figure 1. Schematic diagram of the flow.

complex phenomena. Therefore, in the present work free convective MHD Couette flow of a viscoelastic dusty fluid in a rotating frame is considered and the velocity is taken as complex velocity for both the fluid and the dust particles. This complex velocity is basically the combination of primary velocity in $x$-direction while the secondary velocity is in $z$-direction due to the fact that the rotation is considered about $y$-axis.

\section{Mathematical modeling}

In this work, an unsteady, incompressible viscoelastic fluid with spherical shaped embedded dust particles in a rotating frame is considered. The infinitely rigid plate is extended in $x$-direction and $z$-direction. It is assumed, that motion of the fluid is along $x$-direction, which cover the $x z$-plane at $y \geq 0$. The subject fluid is electrically conducted and uniform magnetic field $B_{0}$ is applied transversely to the flow direction. The system is considered to be in solid body rotation with a uniform angular velocity $\Omega$. The effect of heat transfer with thermal radiation is also taken into account. Initially, at lower plate the ambient temperature $T_{\infty}$ has been considered. At $t=0^{+}$, the upper plate starts oscillation, which generates momentum in the fluid, this momentum is enhanced with impact of wall temperature $T_{w}$ as shown in Fig. 1.

The velocity and the temperature fields for the above flow regime are

$$
\begin{gathered}
\vec{V}=(u(y, t), \Omega, w(y, t)), \\
T=(T(y, t), 0,0) .
\end{gathered}
$$

The basic constitutive equations for the rotating flow of viscoelastic fluid are given as:

$$
\begin{gathered}
\nabla \cdot \vec{V}=0 \\
\rho\left[\frac{d \vec{V}}{d t}+2(\Omega \times \vec{V})+\Omega \times(\Omega \times \vec{r})\right]=\operatorname{div} \overrightarrow{\mathbf{T}}+\rho \vec{b}+\vec{s},
\end{gathered}
$$

where $\mathrm{T}$ is the Cauchy stress tensor and defined as:

$$
\mathbf{T}=-P \mathbf{I}+\mu \mathbf{A}_{1}+\alpha_{1} \mathbf{A}_{2}+\alpha_{2} \mathbf{A}_{1}^{2},
$$

$\mathrm{P}$ is the pressure, $\mathrm{I}$ is the identity matrix, $\mu$ is the viscosity of the fluid, $\alpha_{1}, \alpha_{2}$ are material parameters, $A_{1}, A_{2}$ are Revillon Erickson tensors of first and second kind respectively, defined as:

$$
\begin{aligned}
& \mathbf{A}_{1}=\mathbf{L}+\mathbf{L}^{T}, \\
& \mathbf{A}_{2}=\frac{d \mathbf{A}_{1}}{d t}+\mathbf{A}_{1} \mathbf{L}+\mathbf{L}^{T} \mathbf{A}_{1} .
\end{aligned}
$$

$\rho \vec{b}$ are body forces which includes Lorentz forces and bouncy forces, $\vec{s}$ is the surface interactive forces that generates due to the interaction between dust particles and fluid particles and defined as:

$$
\begin{aligned}
& \rho \vec{b}=J \times B+\rho g \beta_{T}\left(T-T_{\infty}\right), \\
& \vec{s}=K_{0} N_{0}\left(\vec{W}_{1}-\vec{V}\right) .
\end{aligned}
$$

Keeping in mind Eq. (1) and using of Eqs. (5-7), Eq. (4) can be written in component form as ${ }^{44-47}$ : 


$$
\begin{gathered}
\frac{\partial u(y, t)}{\partial t}+2 \Omega w(y, t)=\left(v+\frac{\alpha_{1}}{\rho} \frac{\partial}{\partial t}\right) \frac{\partial^{2} u(y, t)}{\partial y^{2}}+\frac{K_{0} N_{0}}{\rho}\left(w_{1}(y, t)-u(y, t)\right)-\frac{\sigma B_{0}^{2} u}{\rho}+g_{x} \beta_{T}\left(T-T_{\infty}\right), \\
\frac{\partial w(y, t)}{\partial t}-2 \Omega u(y, t)=\left(v+\frac{\alpha_{1}}{\rho} \frac{\partial}{\partial t}\right) \frac{\partial^{2} w(y, t)}{\partial y^{2}}+\frac{K_{0} N_{0}}{\rho}\left(w_{2}(y, t)-w(y, t)\right)-\frac{\sigma B_{0}^{2} w(y, t)}{\rho} \\
m \frac{\partial w_{1}(y, t)}{\partial t}+2 \Omega w_{2}(y, t)=K_{0}\left(u(y, t)-w_{1}(y, t)\right) \\
m \frac{\partial w_{2}(y, t)}{\partial t}-2 \Omega w_{1}(y, t)=K_{0}\left(w(y, t)-w_{2}(y, t)\right) \\
\frac{\partial T(y, t)}{\partial t}=\frac{k}{\rho c_{p}} \frac{\partial^{2} T(y, t)}{\partial y^{2}}-\frac{\partial q_{r}(y, t)}{\partial y}
\end{gathered}
$$

where $\frac{\partial q_{r}}{\partial y}$ is radiation for optically thin fluid and its value is approximated as ${ }^{48}$

$$
-\frac{\partial q_{r}}{\partial y}=4 \alpha_{0}^{2}\left(T-T_{\infty}\right)
$$

The physical initial and boundary conditions are:

$$
\begin{aligned}
& u(y, t)=w(y, t)=0 ; \quad \text { at } t=0, y>0, \\
& \left.\begin{array}{l}
u(y, t)=w(y, t)=0 ; \quad \text { at } y=0, \\
u(y, t)=u_{0} \operatorname{Im} e^{i \omega t}, w(y, t)=0 ; \quad y=d,
\end{array}\right\} t>0
\end{aligned}
$$

where $u, w, w_{1}, w_{2}, v, \alpha_{1}, \rho, K_{0}, N_{0}, \sigma, B_{0}, g, \beta_{T}, T, k, \alpha_{0}$ and $C_{p}$ are primary velocity of the fluid, secondary velocity of the fluid, primary velocity of dust particle, primary velocity of dust particle, kinematic viscosity, material parameter, fluid density, stocks resistance coefficient, number density of the dust particles, electrical conductivity, applied magnetic field, applied magnetic field, gravitational acceleration, coefficient of thermal expansion, temperature of the fluid, thermal conductivity, mean radiation coefficient and specific heat capacity of the fluid respectively. Equations (8-11) represents primary and secondary velocity of the fluid and dust particles respectively. The complex velocities for fluid phase and dust phase have obtained from Eqs. $(8,9)$ and $(10,11)$, respectively

$$
\begin{gathered}
\frac{\partial F(y, t)}{\partial t}-2 i \Omega F(y, t)=\left(v+\frac{\alpha_{1}}{\rho} \frac{\partial}{\partial t}\right) \frac{\partial^{2} F(y, t)}{\partial y^{2}}+\frac{K_{0} N_{0}}{\rho}(W(y, t)-F(y, t))-\frac{\sigma B_{0}^{2} F(y, t)}{\rho}+g \beta_{T}\left(T-T_{\infty}\right) \\
m \frac{\partial W(y, t)}{\partial t}-2 i \Omega W(y, t)=K_{0}(F(y, t)-W(y, t)) \\
\frac{\partial T(y, t)}{\partial t}=\frac{k}{\rho c_{p}} \frac{\partial^{2} T(y, t)}{\partial y^{2}}+4 \alpha_{0}^{2}\left(T-T_{\infty}\right)
\end{gathered}
$$

where, $g=g_{x}+g_{y}$ and $g_{y}=0, F(y, t)=u(y, t)+i w(y, t), W(y, t)=w_{1}(y, t)+i w_{2}(y, t)$ are the complex velocities of fluid and dust particles with the transform initial and boundaries conditions:

$$
\begin{array}{ll}
F(0, t)=0, & T(0, t)=T_{\infty} y=0, t>0, \\
F(d, t)=F_{0} \operatorname{Im} e^{i \omega t}, & T(d, t)=T_{w}, y=d, t>0,
\end{array}
$$

Assume the solution of Eq. (12) obtained through Poincare-Light Hill Technique ${ }^{49}$

$$
W(y, t)=w_{0}(y) e^{i \omega t}
$$

Then the complex velocity $W$ of dust particles in terms of complex velocity $F$ of fluid will be

$$
W(y, t)=\left(\frac{K_{0}}{m i \omega-2 i \Omega m+K_{0}}\right) F(y, t),
$$

By incorporating $W$ in Eq. (14) we get:

$$
\begin{aligned}
\frac{\partial F(y, t)}{\partial t}-2 i \Omega F(y, t)= & \left(v+\frac{\alpha_{1}}{\rho} \frac{\partial}{\partial t}\right) \frac{\partial^{2} F(y, t)}{\partial y^{2}}+\frac{K_{0} N_{0}}{\rho}\left(\frac{K_{0}}{m i \omega-2 i \Omega m+K_{0}}-1\right) F(y, t) \\
& -\frac{\sigma B_{0}^{2} F(y, t)}{\rho}+g \beta_{T}\left(T-T_{\infty}\right)
\end{aligned}
$$




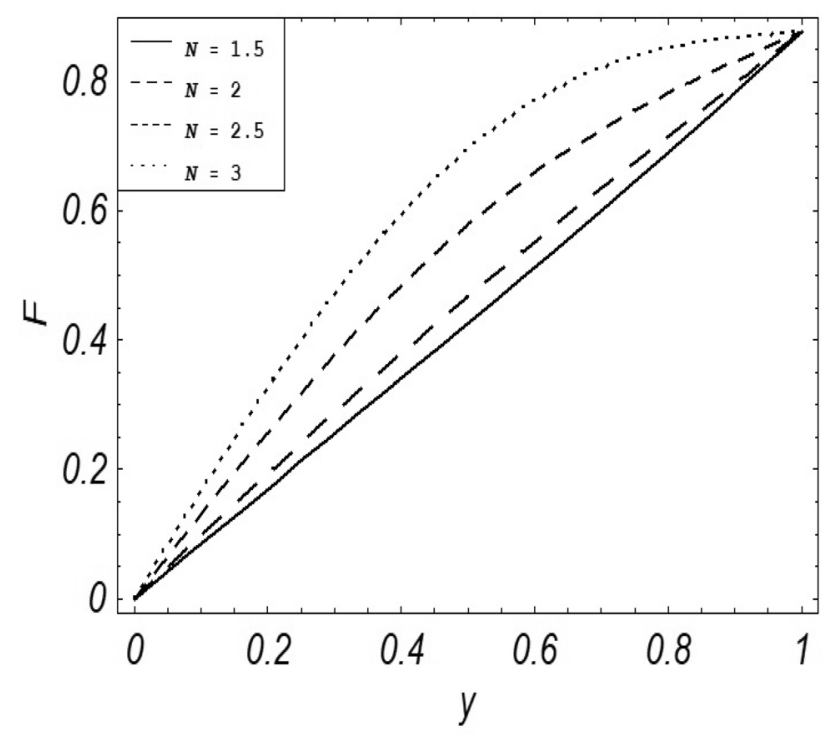

Figure 2. Evolution of $F$ with various values of $N$ if $t=1 ; G r=2.5 ; K_{1}=0.5 ; \eta=2 ; M=0.2 ; \Omega=0.5$; $\operatorname{Re}=1 ; \alpha=0.1 ; \omega=0.5 ; \varepsilon=0.001$.

Introducing the dimensionless variables derived by using Buckingham-Pi theorem;

$$
F^{*}=\frac{F}{F_{0}}, \quad y *=\frac{y}{d}, \quad t *=\frac{F_{0} t}{d}, \quad \theta=\frac{T-T_{\infty}}{T_{w}-T_{\infty}}, \quad \tau^{*}=\frac{\tau d^{2}}{\mu v},
$$

using Eq. (21) , Eq. (16), Eq. (17) and Eq. (20) become dimensionless of the form:

$$
\begin{aligned}
\operatorname{Re} \frac{\partial F(y, t)}{\partial t}-2 i \eta F(y, t)=\frac{\partial^{2} F(y, t)}{\partial y^{2}}+\alpha \frac{\partial^{3} F(y, t)}{\partial t \partial y^{2}}+\left(K_{2}-K_{1}\right) F(y, t)-M F(y, t)+G r \theta(y, t) & \\
P e \frac{\partial \theta(y, t)}{\partial t} & =\frac{\partial^{2} \theta(y, t)}{\partial y^{2}}+N^{2} \theta(y, t) \\
F(0, t) & =0, \quad \theta(0, t)=0, \\
F(1, t) & =\operatorname{Im} e^{i \omega t}, \theta(1, t)=1,
\end{aligned}
$$

where

$$
\begin{aligned}
& \operatorname{Re}=\frac{F_{0} d}{v}, \quad \alpha=\frac{\alpha_{1} F_{0}}{\rho v d}, \quad K_{1}=\frac{K_{0} N_{0} d^{2}}{\rho v}, \quad K_{2}=\frac{K_{0}^{2} N_{0} d^{2}}{\rho v\left(m i \omega-2 i \Omega m+K_{0}\right)}, \\
& M=\frac{\sigma B_{0}^{2} d^{2}}{\rho v}, \quad G r=\frac{g \beta_{T} d^{2}\left(T_{w}-T_{\infty}\right)}{v F_{0}}, \quad P e=\frac{\rho c_{p} F_{0} d}{k}, \quad \eta=\frac{\Omega d^{2}}{v}, \quad N^{2}=\frac{4 \alpha_{0}^{2} d^{2}}{k}
\end{aligned}
$$

While Re, $\alpha, K_{1}, K_{2}, M, G r, P e, N^{2}$ and $\eta$ represents the dimensionless Reynolds number, second grade parameter, Dusty parameters, magnetic parameter, Grashof number, Peclet number, radiation parameter and dimensionless rotational parameter respectively.

(Note: To avoid complexity ${ }^{\star}$ has been dropped).

Consider the following assume periodic solutions for energy equation obtained through Poincare-Light Hill Technique ${ }^{49}$

$$
\theta(y, t)=\theta_{0}(y)+\varepsilon \theta_{1}(y) e^{i \omega t}+O\left(\varepsilon^{2}\right) .
$$

By solving energy equation with the help of above assumed periodic solution and ignoring higher order of $\varepsilon$ we get;

$$
\theta_{0}(y)=\frac{\operatorname{Sin}(N y)}{\operatorname{Sin} N} ; \theta_{1}(y)=0 .
$$

Using the values of $\theta_{0}(y)$ and $\theta_{1}(y)$ in Eq. (26), then the final solution of energy equation will be: 


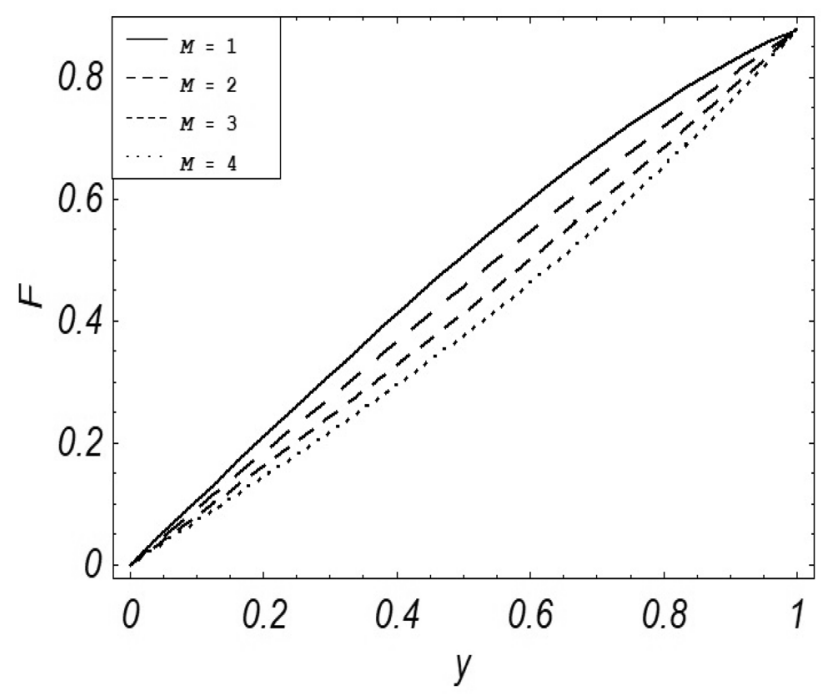

Figure 3. Evolution of $F$ with various values of $M$ if $t=1 ; G r=2.5 ; N=0.7 ; K=0.5 ; \eta=2 \Omega=0.5$; $\operatorname{Re}=1 ; \alpha=0.1 ; \omega=0.5 ; \varepsilon=0.001$.

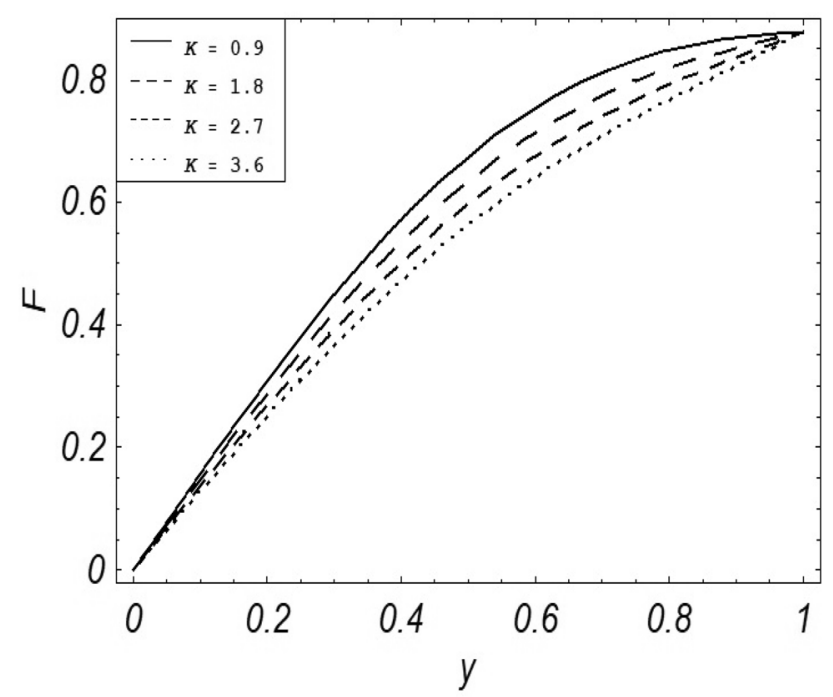

Figure 4. Evolution of $F$ with various values of $K$ if $t=1 ; G r=2.5 ; N=0.7 ; \eta=2 ; G r=2.5 \Omega=0.5$; $\operatorname{Re}=1 ; \alpha=0.1 ; \omega=0.5 ; \varepsilon=0.001$.

$$
\theta(y, t)=\frac{\operatorname{Sin}(N y)}{\operatorname{Sin} N}
$$

Now by coupling energy in momentum equation and assuming the periodic solution obtained through Poincare-Light Hill Technique ${ }^{49}$

$$
F(y, t)=F_{1}(y)+\varepsilon F_{2}(y) e^{i \omega t}+O\left(\varepsilon^{2}\right)
$$

Incorporating the above assume solution in Eq. (22), and separating the harmonic and non-harmonic parts, the following values for $F_{1}(y)$ and $F_{2}(y)$ are obtained

$$
F_{1}(y)=A\left\{\frac{\operatorname{Sin}(N y)}{\operatorname{Sin}(N)}-\frac{\operatorname{Sinh}\left(\sqrt{M_{1}} y\right)}{\operatorname{Sinh}\left(\sqrt{M_{1}}\right)}\right\}, \quad F_{2}(y)=\frac{\operatorname{Sinh}\left(\sqrt{M_{2}} y\right)}{\varepsilon \operatorname{Sinh}\left(\sqrt{M_{2}}\right)},
$$




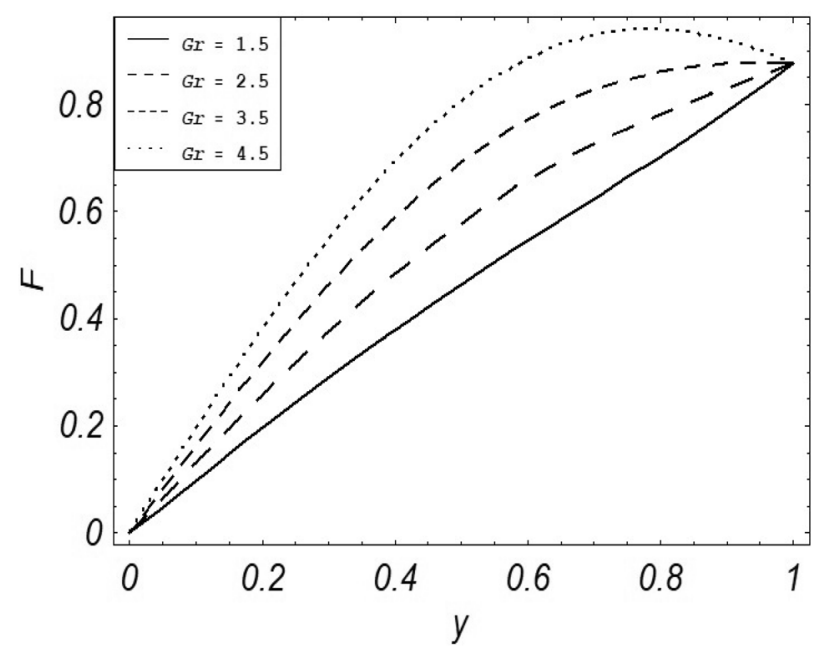

Figure 5. Evolution of $F$ with various values of $G r$ if $t=1 ; N=0.7 ; K=0.5 ; \eta=2 ; N=1 \Omega=0.5$; $\operatorname{Re}=1 ; \alpha=0.1 ; \omega=0.5 ; \varepsilon=0.001$.

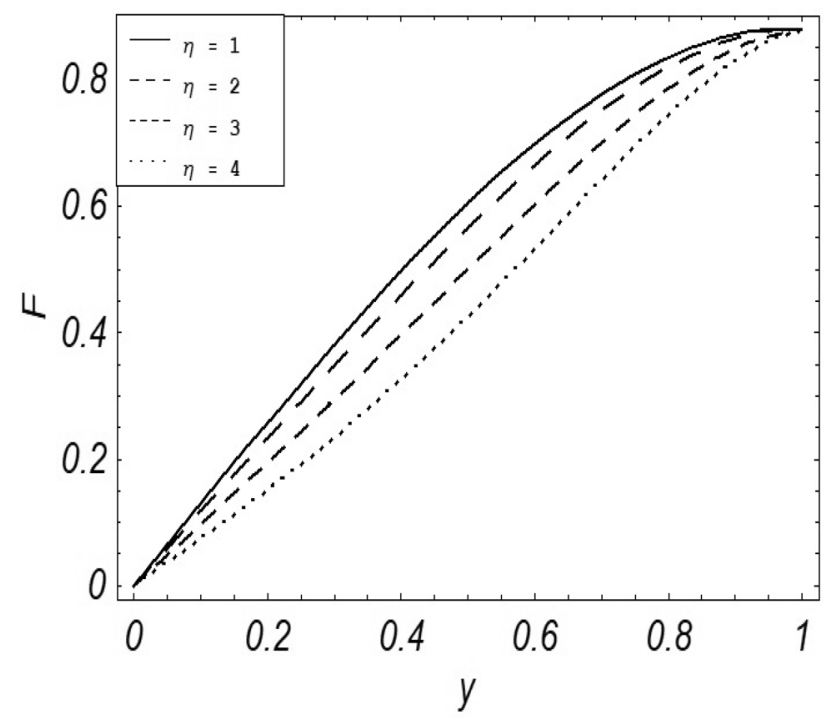

Figure 6. Evolution of $F$ with various values of $\eta$ if $t=1 ; G r=2.5 ; K_{1}=0.5 ; N=1 ; M=0.2 ; \Omega=0.5$; $\operatorname{Re}=1 ; \alpha=0.1 ; \omega=0.5 ; \varepsilon=0.001$.

$$
F(y, t)=A\left\{\frac{\operatorname{Sin}(N y)}{\operatorname{Sin}(N)}-\frac{\operatorname{Sinh}\left(\sqrt{M_{1}} y\right)}{\operatorname{Sinh}\left(\sqrt{M_{1}}\right)}\right\}+\left\{\frac{\operatorname{Sinh}\left(\sqrt{M_{2}} y\right)}{\operatorname{Sinh}\left(\pi \sqrt{M_{2}}\right)}\right\} e^{i \omega t} .
$$

where

$$
A=\frac{G r}{N^{2}+M_{1}}, \quad M_{1}=K_{2}-K_{1}-2 i \eta, \quad M_{2}=\frac{K_{2}-K_{1}+M+\operatorname{Re} i \omega t-2 i \eta}{1+\alpha i \omega} .
$$

\section{Special case}

By considering $\eta \rightarrow 0$ and $G r \rightarrow 0$ the general solution which reduces Eq. (31) to the following form:

$$
u(y, t)=e^{i \omega t}\left(\frac{\sinh \sqrt{M_{4}} y}{\sinh \sqrt{M_{4}}}\right)
$$

which represents Couette flow of electrically conducting viscoelastic dusty fluid. 


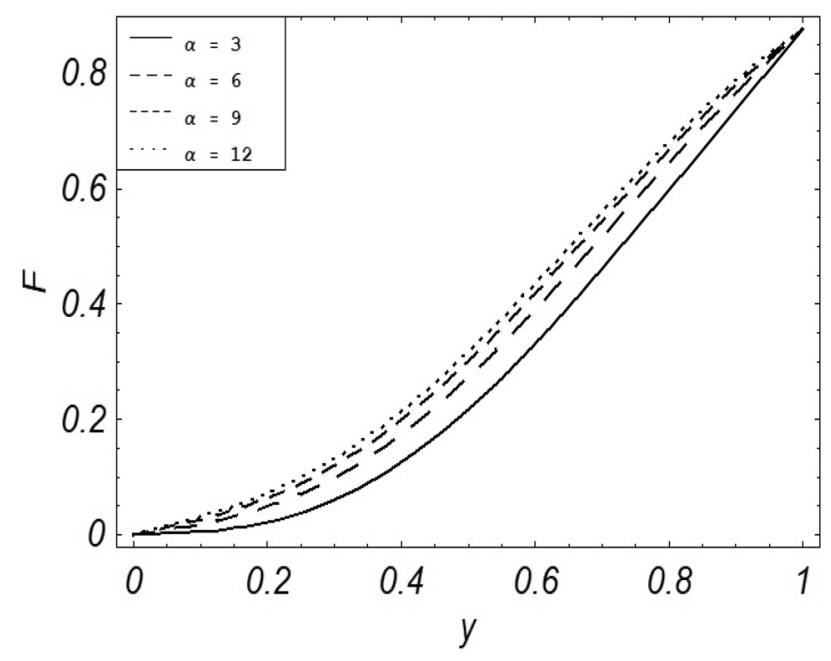

Figure 7. Evolution of $F$ with various values of $\alpha$ if $t=1 ; G r=2.5 ; N=0.7 ; K=0.5 ; \eta=2 \Omega=0.5$; $\operatorname{Re}=1 ; M=0.1 ; \omega=0.5 ; \varepsilon=0.001$.

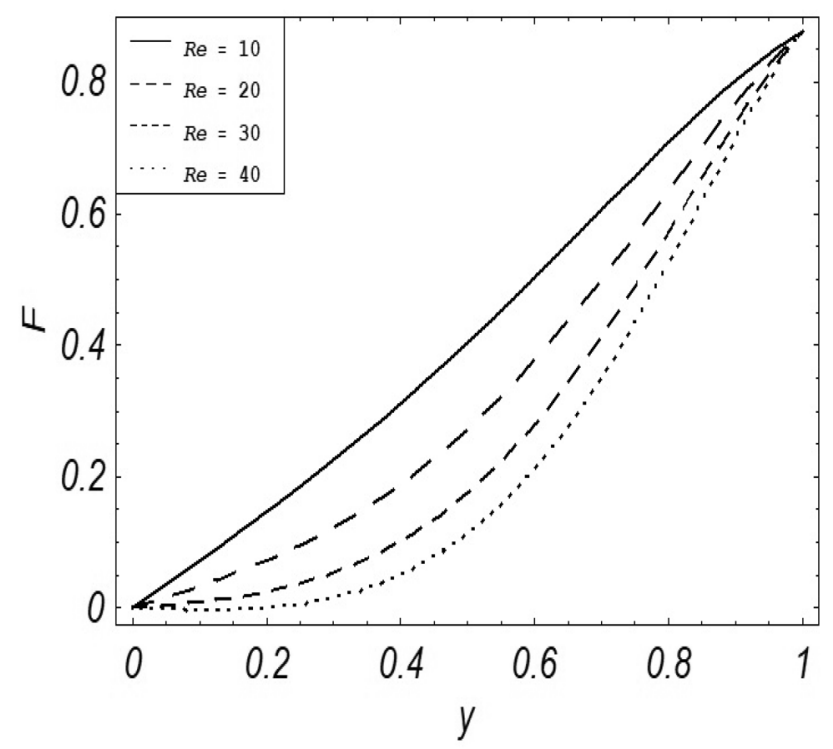

Figure 8. Evolution of $F$ with various values of Re if $t=1 ; G r=2.5 ; N=0.7 ; \eta=2 ; G r=2.5$ $\Omega=0.5 ; K=1 ; \alpha=0.1 ; \omega=0.5 ; \varepsilon=0.001$.

\section{Nusselt number}

Nusselt number is a dimensionless number which is the ratio of convective to conductive heat transfer at a boundary in a fluid. The dimensional form of the Nusselt number is given as:

$$
N u=\left.\frac{d}{\left(T_{w}-T_{\infty}\right)} \frac{\partial T}{\partial y}\right|_{y=0} .
$$

The dimensionless Nusselt number is presented by

$$
N u=\left.\frac{\partial \theta}{\partial y}\right|_{y=0}=\frac{N}{\operatorname{Sin}[N]},
$$




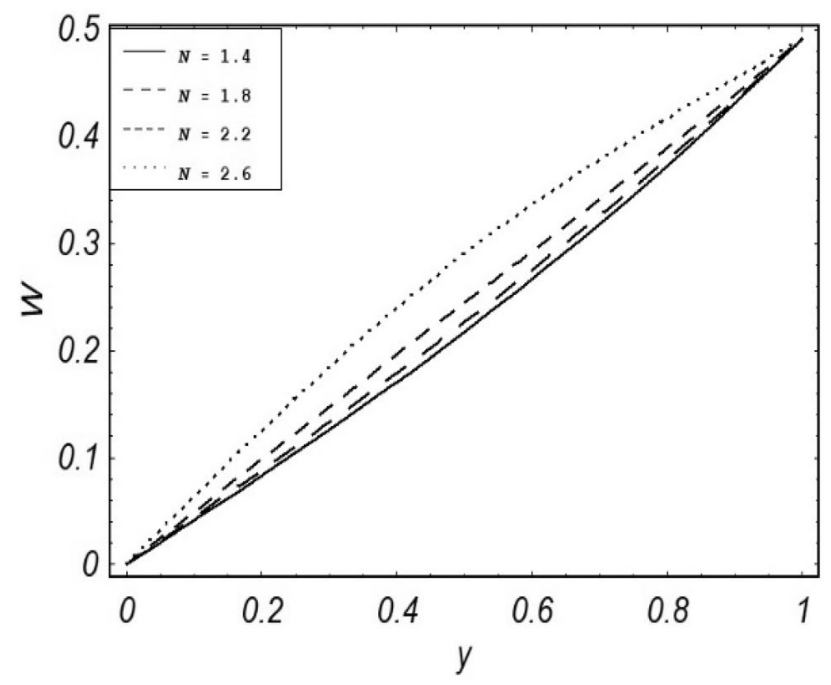

Figure 9. Evolution of $W$ with various values of $N$ if $t=1 ; G r=0.7 ; K=0.5 ; \eta=2 ; \Omega=0.5$; $\operatorname{Re}=1 ; \alpha=0.1 ; \omega=0.5 ; \varepsilon=0.001 ; m=0.5$.

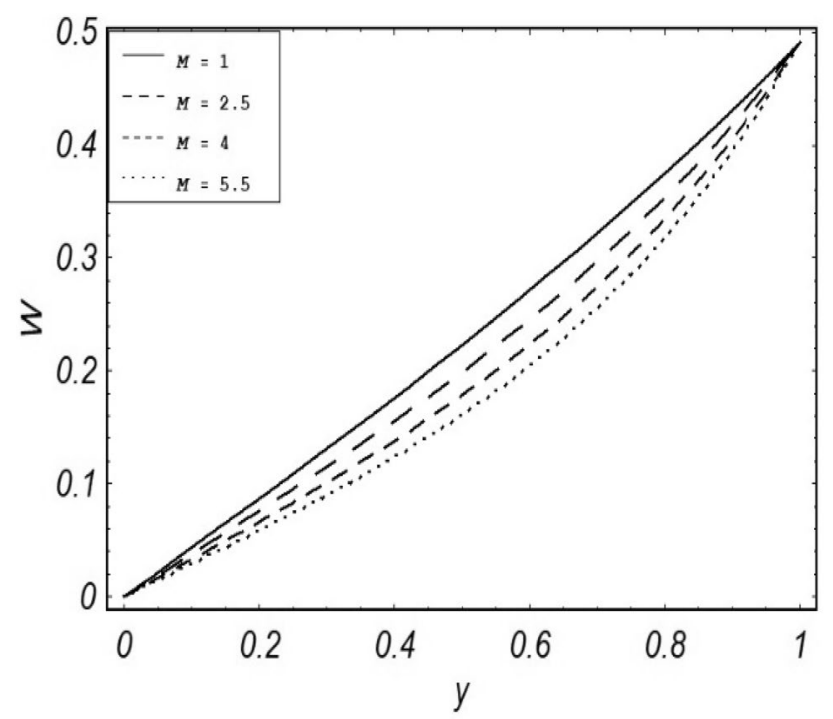

Figure 10. Evolution of $W$ with various values of $M$ if $t=1 ; G r=2.5 ; K_{1}=0.5 ; N=1 ; \eta=0.2 ; m=0.5$; $\Omega=0.5 ; \operatorname{Re}=1 ; \alpha=0.1 ; \omega=0.5 ; \varepsilon=0.001$.

\section{Skin friction}

The Drag force which is generated by the friction of fluid against the lower plate's surface at $y=0$ that is moving through is called skin friction. As the subject fluid is non-Newtonian viscoelastic, so the expression for the skin friction is given by

$$
\tau=\left(\mu+\alpha_{1} \frac{\partial}{\partial t}\right) \frac{\partial F}{\partial y} .
$$

Using Eq. (21) in the above equation the following dimensionless form for skin friction is obtained

$$
\tau=\operatorname{Re} \frac{\partial F}{\partial y}+\alpha \frac{\partial^{2} F}{\partial t \partial y} .
$$

Incorporating Eq. (31) in Eq. (34) the following expression for skin friction has been obtained

$$
\tau=\frac{-A \operatorname{Re} \sqrt{M_{1}}}{\operatorname{Sinh} \sqrt{M_{1}}}+\frac{e^{i \omega t}(\operatorname{Re}+i \omega) \sqrt{M_{2}}}{\operatorname{Sinh} \sqrt{M_{2}}} .
$$




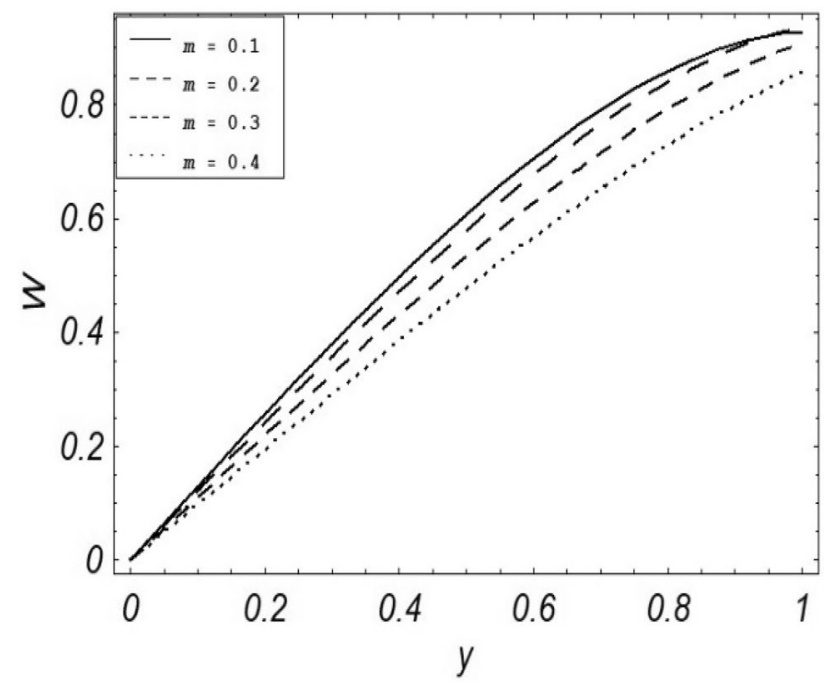

Figure 11. Evolution of $W$ with various values of $m$ if $t=1 ; G r=2.5 ; N=0.7 ; K=0.5 ; \eta=2 \Omega=0.5$; $\mathrm{Re}=1 ; M=0.1 ; \omega=0.5 ; \varepsilon=0.001 ; \alpha=0.5$.

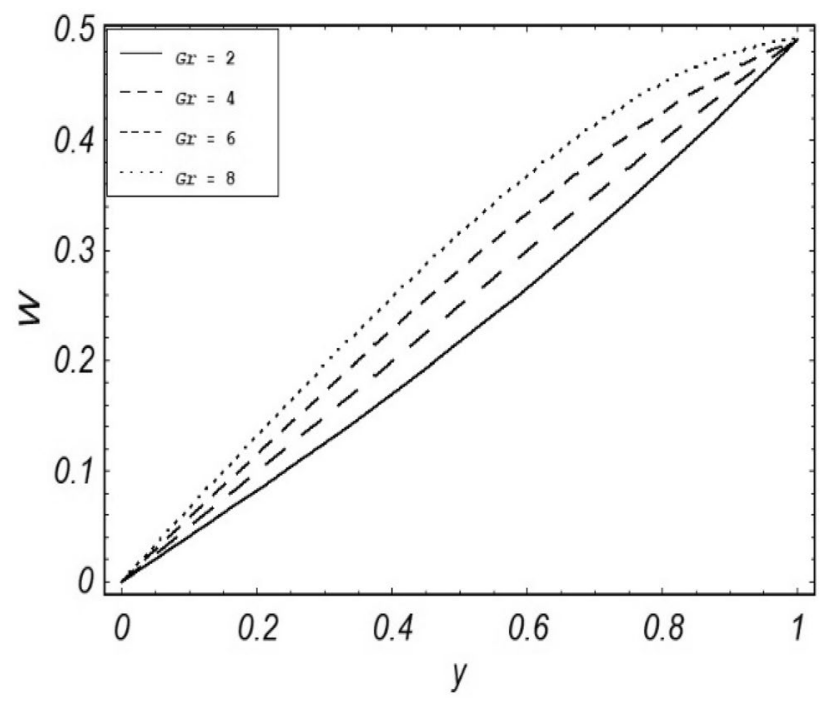

Figure 12. Evolution of $W$ with various values of $G r$ if $t=1 ; M=2.5 ; K_{1}=0.5 ; N=1 ; \eta=0.2 ; m=0.5$; $\Omega=0.5 ; \operatorname{Re}=1 ; \alpha=0.1 ; \omega=0.5 ; \varepsilon=0.001$.

\section{Discussion and graphical results}

In this article the effect of various physical dimensionless parameters on velocity profile of the base fluid as well as the velocity profile of dust particles has been discussed. The changes in skin friction and Nusselt number with these physical parameters have also been analyzed.

Figures 2, 3, 4, 5, 6, 7 and 8 represent the behavioral changes in velocity profile of the fluid by varying different physical parameters like Magnetic parameter $M$, Reynolds number Re, Second-grade parameter $\propto$, Radiation parameter N, Dusty fluid parameter K, Rotational parameter $\eta$, Grashoff Number Gr. While Figs. 9, 10, 11, 12, 13, 14 and 15 are presenting the behavioral changes of the dust particle's velocity with the variation of all the above mentioned physical parameters. The variation of the velocity profile of dust particles is also analyzed by varying mass $m$ of the dust particle. In Fig. 16 the effect on temperature with the radiation parameter $N$ is shown.

It is noticed that by increasing $M$ considerably retards the velocity of the fluid as well as the dust particles. By escalating $M$, Lorentz forces are enhanced which enlarges the resistive forces present inside of a fluid, due to the increase of these resistive forces the velocity of the fluid phase and dusty phase retards. Gr is the ratio of buoyancy forces to viscous forces hence increasing the values of $\mathrm{Gr}$ increases the buoyancy forces and decreases the viscous forces and as a result accelerates the velocities of the fluid and the dust particles. We have considered laminar flow of a viscoelastic dusty fluid. In laminar flow viscous forces are significant. Re is the ratio of inertial forces to viscous forces, therefore by increasing the Reynolds number inertial forces are increased which retards 


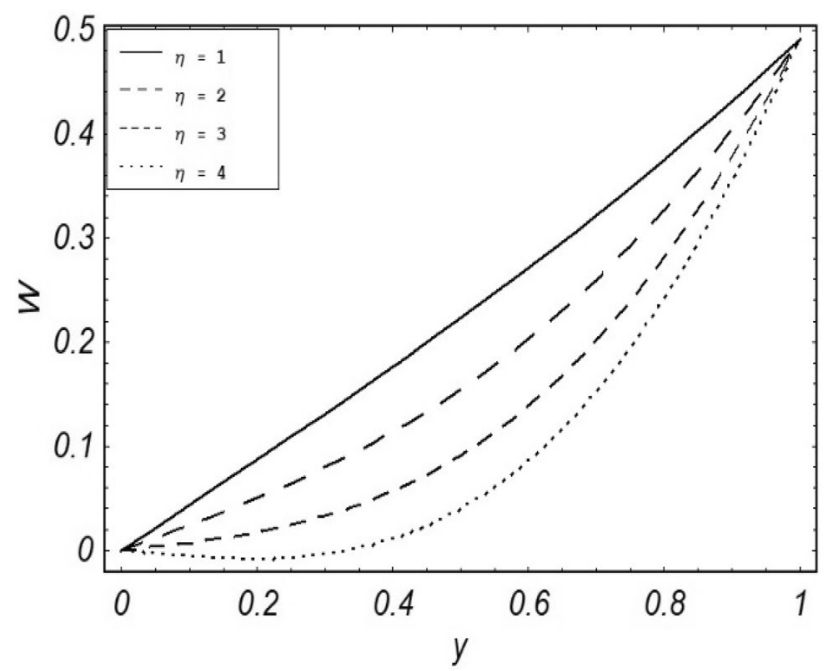

Figure 13. Evolution of $W$ with various values of $\eta$ if $t=1 ; M=2.5 ; K_{1}=0.5 ; N=1 ; G r=0.2$; $m=0.5 ; \Omega=0.5 ; \operatorname{Re}=1 ; \alpha=0.1 ; \omega=0.5 ; \varepsilon=0.001$.

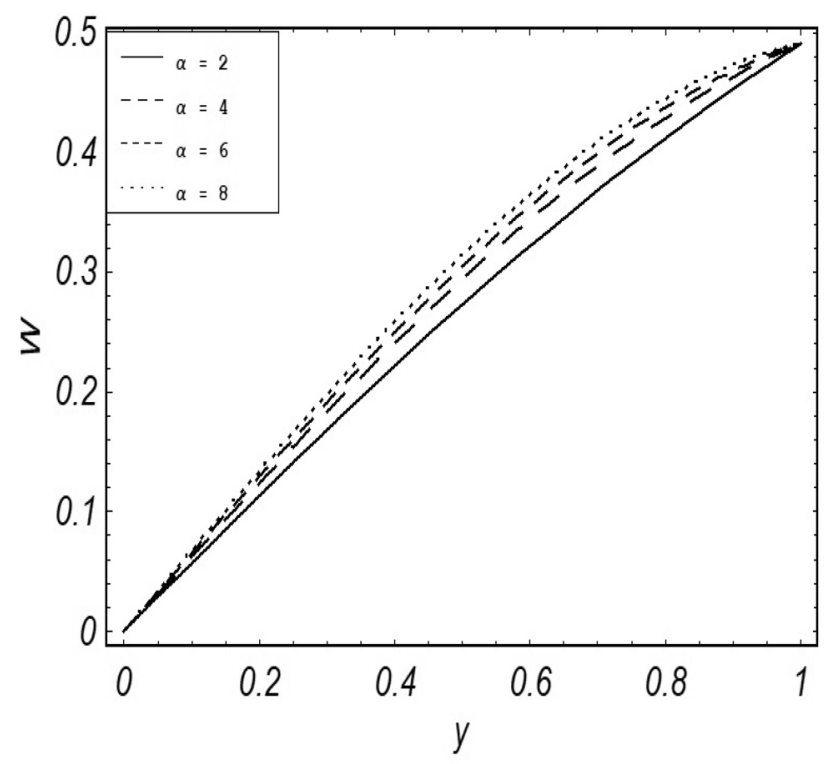

Figure 14. Evolution of $W$ with various values of $\alpha$ if $t=1 ; G r=2.5 ; K=0.5 ; N=1 ; \eta=0.2 ; m=0.5$; $\Omega=0.5 ; \operatorname{Re}=1 ; M=0.5 ; \omega=0.5 ; \varepsilon=0.001$.

the flow. Rotational parameter $\eta=\Omega d^{2} / v$ causes retardation in the velocities of dust particles and the fluid due to the fact that the increase in $\eta$ enhances the Coriolis forces that are in fact inertial forces. Moreover, increasing $\eta$ decreases $v$ where $v=\mu / \rho$ hence with the decrease of viscous forces inertial forces are enhanced, making inertial forces strong enough to retard the velocities of the fluid phase and the dust phase. It is evident from Figs. 2 and 9 that the increase in radiation parameter $N$ escalates the velocity of both the fluid phase and the dust phase. This is due to the fact that $N$ has a direct relation with temperature which increases the internal kinetic energy of the subject fluid. Since in this article the dust particles are assumed to be in a spherical shape and are homogeneously distributed throughout the subject fluid. According to the Stokes Law drag force $F_{d}=6 \pi \mu r$ hence by increasing concentration of dust particles $\mathrm{K}$, will increase this drag force which in turn increases the viscosity of the fluid rendering to the decrease in the velocity of the fluid phase and the dust phase. Figure 11 shows retarding behavior of the velocity of the dust particles with the increasing value of mass $m$ because of the fact that force and mass have a direct relation, so by increasing the mass $m$ will increase the drag force which increases the viscosity rendering a retarding effect on the velocity of the dust particles. Figure 16 shows the relation between radiation parameter $N$ and temperature, due to the fact that kinetic energy is increased due to an increase in $N$ which enhances the temperature of the fluid ("Supplememtary Information"). 


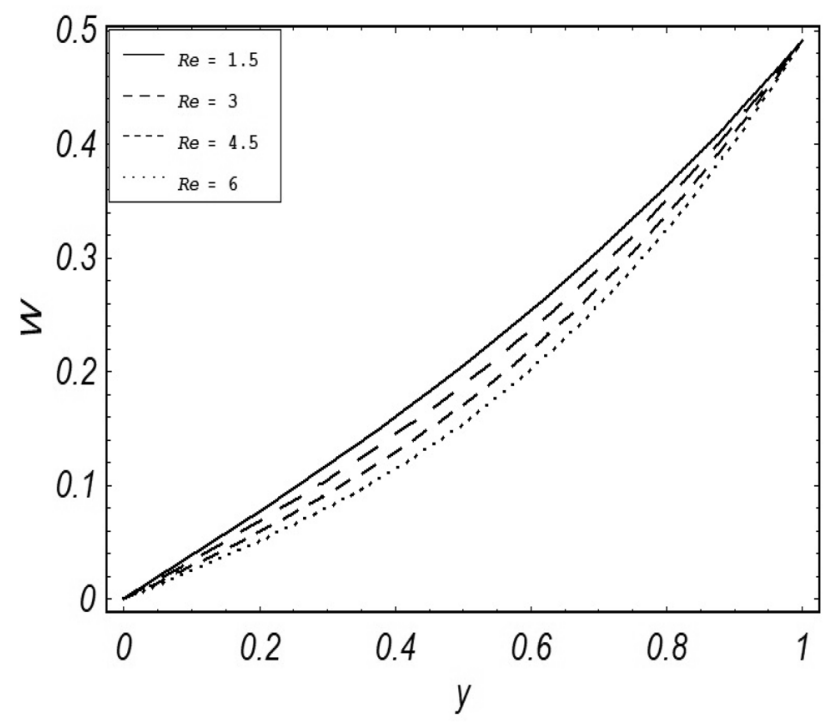

Figure 15. Evolution of $W$ with various values of Re if $t=1 ; G r=2.5 ; N=0.7 ; K=0.5 ; \eta=2 \Omega=0.5$; $m=1 ; M=0.1 ; \omega=0.5 ; \varepsilon=0.001 ; \alpha=0.5$.

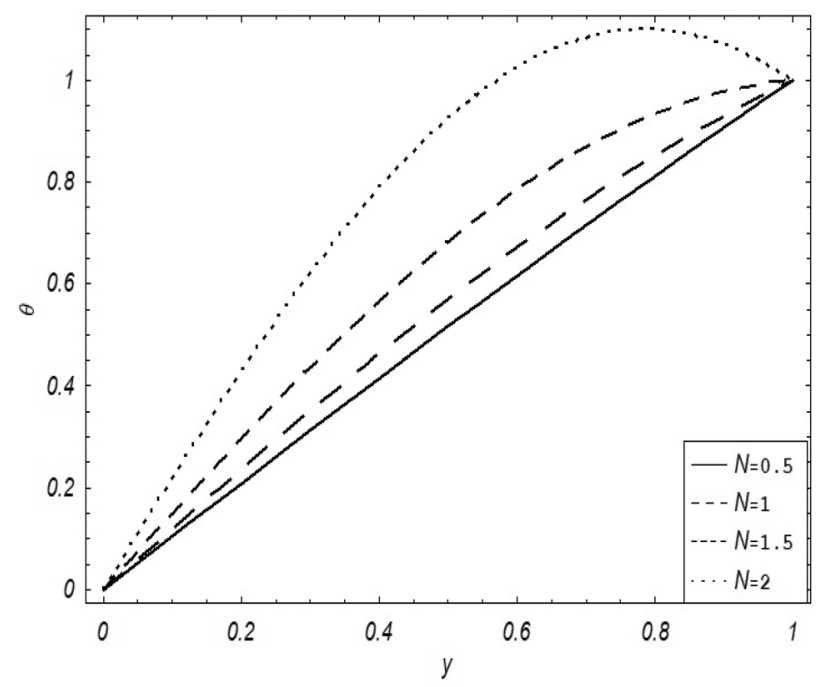

Figure 16. Variation of temperature with $N$.

\begin{tabular}{|l|l|l|l|l|l|l|l|l|l|}
\hline$\eta$ & $\boldsymbol{t}$ & $\boldsymbol{\omega}$ & $\boldsymbol{K}$ & $\boldsymbol{N}$ & $\boldsymbol{M}$ & $\boldsymbol{\alpha}$ & $\mathbf{R e}$ & $\boldsymbol{G r}$ & $\boldsymbol{C} \cdot \boldsymbol{f}$ \\
\hline 0.5 & 1 & $0.5 \pi$ & 0.5 & $\mathbf{1 . 5}$ & 0.2 & 0.1 & 5 & 0.5 & 1.3149 \\
\hline 0.5 & 1 & $0.5 \pi$ & 0.5 & $\mathbf{2}$ & 0.2 & 0.1 & 5 & 0.5 & 1.2595 \\
\hline 0.5 & 1 & $0.5 \pi$ & 0.5 & $\mathbf{2 . 5}$ & 0.2 & 0.1 & 5 & 0.5 & 1.1736 \\
\hline
\end{tabular}

Table 1. Influence of radiation parameter $N$ on skin friction.

\begin{tabular}{|l|l|l|l|l|l|l|l|l|l|}
\hline $\boldsymbol{\eta}$ & $\boldsymbol{t}$ & $\boldsymbol{\omega}$ & $\boldsymbol{C} . \boldsymbol{f}$ & $\boldsymbol{N}$ & $\boldsymbol{M}$ & $\boldsymbol{\alpha}$ & $\mathrm{Re}$ & $\mathbf{G r}$ & $\boldsymbol{C} \cdot \boldsymbol{f}$ \\
\hline 0.5 & 1 & $0.5 \pi$ & 0.2 & 0.5 & $\mathbf{1}$ & 3 & 0.5 & 10 & 0.60365 \\
\hline 0.5 & 1 & $0.5 \pi$ & 0.2 & 0.5 & $\mathbf{2}$ & 3 & 0.5 & 10 & 0.65838 \\
\hline 0.5 & 1 & $0.5 \pi$ & 0.2 & 0.5 & $\mathbf{3}$ & 3 & 0.5 & 10 & 0.67944 \\
\hline
\end{tabular}

Table 2. Influence of magnetic parameter $M_{\mathrm{on}}$ skin friction. 


\begin{tabular}{|l|l|l|l|l|l|l|l|l|l|}
\hline $\boldsymbol{\eta}$ & $\boldsymbol{t}$ & $\boldsymbol{\omega}$ & $\boldsymbol{C} . \boldsymbol{f}$ & $\boldsymbol{N}$ & $\boldsymbol{M}$ & $\boldsymbol{\alpha}$ & $\mathrm{Re}$ & $\boldsymbol{G r}$ & $\boldsymbol{C} \cdot \boldsymbol{f}$ \\
\hline 0.5 & 1 & $0.5 \pi$ & $\mathbf{0 . 9}$ & 0.5 & 10 & 3 & 0.5 & 10 & 0.60365 \\
\hline 0.5 & 1 & $0.5 \pi$ & $\mathbf{1 . 8}$ & 0.5 & 10 & 3 & 0.5 & 10 & 0.60956 \\
\hline 0.5 & 1 & $0.5 \pi$ & $\mathbf{2 . 7}$ & 0.5 & 10 & 3 & 0.5 & 10 & 0.61655 \\
\hline
\end{tabular}

Table 3. Influence of dusty fluid parameter C.f on skin friction.

\begin{tabular}{|l|l|l|l|l|l|l|l|l|l|}
\hline $\boldsymbol{\eta}$ & $\boldsymbol{t}$ & $\boldsymbol{\omega}$ & $\boldsymbol{C . f}$ & $\boldsymbol{N}$ & $\boldsymbol{M}$ & $\boldsymbol{\alpha}$ & $\mathbf{R e}$ & $\mathbf{G r}$ & $\boldsymbol{C} \cdot \boldsymbol{f}$ \\
\hline 1 & 1 & $0.5 \pi$ & 0.5 & 0.1 & 0.2 & 0.1 & 5 & $\mathbf{1 . 5}$ & 1.3149 \\
\hline 1 & 1 & $0.5 \pi$ & 0.5 & 0.1 & 0.2 & 0.1 & 5 & $\mathbf{2 . 5}$ & 0.6751 \\
\hline 1 & 1 & $0.5 \pi$ & 0.5 & 0.1 & 0.2 & 0.1 & 5 & $\mathbf{3 . 5}$ & 0.00467 \\
\hline
\end{tabular}

Table 4. Influence of Grashof number $G r$ on skin friction.

\begin{tabular}{|l|l|l|l|l|l|l|l|l|l|}
\hline $\boldsymbol{\eta}$ & $\boldsymbol{t}$ & $\boldsymbol{\omega}$ & $\boldsymbol{C . f}$ & $\boldsymbol{N}$ & $\boldsymbol{M}$ & $\boldsymbol{\alpha}$ & $\mathrm{Re}$ & $\mathbf{G r}$ & $\boldsymbol{C} \cdot \boldsymbol{f}$ \\
\hline 1 & 1 & $0.5 \pi$ & 0.2 & 0.5 & 100 & 3 & 0.5 & 10 & 0.60365 \\
\hline 2 & 1 & $0.5 \pi$ & 0.5 & 0.5 & 100 & 3 & 0.5 & 10 & 0.610997 \\
\hline 3 & 1 & $0.5 \pi$ & 0.5 & 0.5 & 100 & 3 & 0.5 & 10 & 0.624351 \\
\hline
\end{tabular}

Table 5. Influence of rotational parameter $\eta$ on skin friction.

\begin{tabular}{|l|l|l|l|l|l|l|l|l|l|}
\hline $\boldsymbol{\eta}$ & $\boldsymbol{t}$ & $\boldsymbol{\omega}$ & $\boldsymbol{C . f}$ & $\boldsymbol{N}$ & $\boldsymbol{M}$ & $\boldsymbol{\alpha}$ & $\mathrm{Re}$ & $\mathbf{G r}$ & $\boldsymbol{C} \cdot \boldsymbol{f}$ \\
\hline 0.5 & 1 & $0.5 \pi$ & 0.2 & 0.5 & 100 & $\mathbf{3}$ & 0.5 & 10 & 0.60365 \\
\hline 0.5 & 1 & $0.5 \pi$ & 0.2 & 0.5 & 100 & $\mathbf{6}$ & 0.5 & 10 & 0.529004 \\
\hline 0.5 & 1 & $0.5 \pi$ & 0.2 & 0.5 & 100 & $\mathbf{9}$ & 0.5 & 10 & 0.31335 \\
\hline
\end{tabular}

Table 6. Influence of second grade parameter $\alpha$ on skin friction.

\begin{tabular}{|l|l|l|l|l|l|l|l|l|l|}
\hline $\boldsymbol{\eta}$ & $\boldsymbol{t}$ & $\boldsymbol{\omega}$ & $\boldsymbol{C} . \boldsymbol{f}$ & $\boldsymbol{N}$ & $\boldsymbol{M}$ & $\boldsymbol{\alpha}$ & $\mathrm{Re}$ & $\boldsymbol{G r}$ & $\boldsymbol{C} \cdot \boldsymbol{f}$ \\
\hline 1 & 1 & $0.5 \pi$ & 0.5 & 0.1 & 100 & 0.1 & $\mathbf{1 0}$ & 0.5 & 0.62331 \\
\hline 1 & 1 & $0.5 \pi$ & 0.5 & 0.1 & 100 & 0.1 & $\mathbf{2 0}$ & 0.5 & 1.3149 \\
\hline 1 & 1 & $0.5 \pi$ & 0.5 & 0.1 & 100 & 0.1 & $\mathbf{3 0}$ & 0.5 & 1.92353 \\
\hline
\end{tabular}

Table 7. Influence of Reynolds number Re on skin friction.

\begin{tabular}{|l|l|l|l|l|}
\hline Radiation parameter $N$ & 0.3 & 0.5 & 0.7 & 0.9 \\
\hline Nusselt number $\mathrm{Nu}$ & 1.01516 & 1.04291 & 1.08659 & 1.14895 \\
\hline
\end{tabular}

Table 8. Variation in Nusselt number with radiation parameter $N$.

The effect of embedded parameters like Magnetic parameter $M$, Reynolds number Re, Second grade parameter $\alpha$, Radiation parameter $N$, Dusty fluid parameter $K$, Rotational parameter $\eta$, Grashoff Number $G r$ on the skin friction is given in Tables 1, 2, 3, 4, 5, 6 and 7. The bold values in each of the tables show the variation in that specific parameter which has shown in that column. It is noticed that by increasing $M, \operatorname{Re}, \mathrm{K}, \eta$ increases the $C f$ due to the fact discussed earlier that these parameters noticeably increases the inertial forces hence causing an increase in the numerical value of $C f$ While a decreasing behavior of $C f$ is observed with the increase of Gr, $\alpha$, $N$, due to the fact that these parameters decreases viscous forces which decreases the $C f$ Table 8 represents the variation of Nusselt $(\mathrm{Nu})$ with increasing value of radiation parameter $N$. The radiation parameter $N$ depicts a direct relation with the Nusselt number that is by increasing $N$ an increase in Nusselt number has been observed. 


\section{Conclusion}

The present article deals with the rotational viscoelastic dusty fluid under the impact of magnetic field $B_{o}$ which is being applied transversely to the fluid flow with free convection and radiation. A detailed parametric influential analysis has been done on the complete system of the fluid. Keeping in view the graphical results and discussions we can summarize the whole analysis in the following key-points;

- $N, G r$, and $\alpha$ shows a direct relationship with the velocity of the fluid phase and the dust phase.

- $M, K, \eta$ and Re shows an inverse relation with the velocity of fluid phase and the dust phase.

- Enlarging mass $m$ of the dust particles contributes to the decrease in the velocity of the dust phase.

- Skin friction shows a decreasing behavior with the escalating values of $N, G r$ and $\alpha$.

- While the opposite behaviour is observed in the case of $M, K, \eta$ and Re i.e. an increase in skin friction is noticed with the greater values of $M, K, \eta$ and Re.

- The temperature profile of the fluid escalates with the greater value of radiation parameter $N$.

- A direct relation between the Nusselt number and radiation parameter $N$ has been noticed.

Received: 4 August 2020; Accepted: 25 November 2020

Published online: 12 January 2021

\section{References}

1. Dey, D. Viscoelastic fluid flow through an annulus with relaxation, retardation effects and external heat source/sink. Alexandria Eng. J. 57(2), 995-1001. https://doi.org/10.1016/j.aej.2017.01.039 (2018).

2. Oldroyd, J. G. On the formulation of rheological equations of state. Proc. R. Soc. Lond. Series A Math. Phys. Sci. 200(1063), 523-541. https://doi.org/10.1098/rspa.1950.0035 (1950).

3. Rajagopal, K. R. \& Bhatnagar, R. K. Exact solutions for some simple flows of an Oldroyd-B fluid. Acta Mech. 113(1-4), 233-239. https://doi.org/10.1007/BF01212645 (1995).

4. Kuiken, H. K. Magnetohydrodynamic free convection in a strong cross field. J. Fluid Mech. 40(1), 21-38. https://doi.org/10.1017/ S0022112070000022 (1970).

5. Michael, D. H. \& Miller, D. A. The plane-parallel flow of the dusty gas. Mathematica 13, 67. https://doi.org/10.1112/S002557930 0004289 (1966).

6. Soo, S. L. Fluid dynamics of the multiphase systems. 524. https://trove.nla.gov.au/work/15284845 (Blaisdell Publishing Company, Waltham, 1967).

7. Venkatesh, P. \& Kumara, B. P. Exact solution of an unsteady conducting dusty fluid flow between the non-torsional oscillating plate and a long wavy wall. J. Sci. Arts 13(1), 97. https://www.icstm.ro/DOCS/josa/josa_2013_1/c_01_Vankatesh_Prasanna.pdf (2013).

8. Ghosh, A. K. \& Sana, P. On hydromagnetic channel flow of an Oldroyd-B fluid induced by rectified sine pulses. Comput. Appl. Math. 28(3), 365-395. https://doi.org/10.1590/S1807-03022009000300006 (2009).

9. Saffman, P. G. On the stability of the laminar flow of the dusty gas. J. Fluid Mech. 13(1), 120-128. https://doi.org/10.1017/S0022 112062000555 (1966).

10. Vimala, C. S. The flow of a dusty gas between two oscillating plates. Defense Sci. J. 22(4), 231-236 (1972).

11. Healy, J. V. Perturbed two-phase cylindrical type flows. Phys. Fluids 13(3), 551-557. https://doi.org/10.1063/1.1692959 (1970).

12. Venkateshappa, V., Rudraswamy, B., Gireesha, B. J. \& Gopinath, K. Viscous dusty fluid flow with constant velocity magnitude. Electron. J. Theor. Phys. 5(17), 237-252 (2008).

13. Gupta, R. K. \& Gupta, S. C. The flow of a dusty gas through a channel with an arbitrary time-varying pressure gradient. Zeitschrift fur Angewandte Math. Phys. ZAMP 27(1), 119-125. https://doi.org/10.1007/BF01595248 (1976).

14. Ghosh, S. \& Ghosh, A. K. On the hydromagnetic rotating flow of a dusty fluid near a pulsating plate. Computat. Appl. Math. 27(1), 1-30. https://www.scielo.br/scielo.php?script=sci_isoref\&pid=S1807-03022008000100001 (2008).

15. Attia, H. A. \& Abdeen, M. A. Steady MHD flow of a dusty incompressible non-Newtonian Oldroyd 8-constant fluid in a circular pipe. Arab. J. Sci. Eng. 38(11), 3153-3160. https://doi.org/10.1007/s13369-012-0475-z (2013).

16. Debnath, L. \& Ghosh, A. K. On unsteady hydromagnetic flows of a dusty fluid between two oscillating plates. Appl. Sci. Res. 45(4), 353-365. https://doi.org/10.1007/BF00457067 (1988).

17. Gireesha, B. J., Chamkha, A. J., Manjunatha, S. \& Bagewadi, C. S. The mixed convective flow of a dusty fluid over a vertical stretching sheet with non-uniform heat source/sink and radiation. Int. J. Numer. Meth. Heat Fluid Flow 23(4), 598-612. https://doi. org/10.1108/09615531311323764 (2013).

18. Ali, F., Imtiaz, A., Khan, I. \& Sheikh, N. A. Flow of magnetic particles in blood with isothermal heating: A fractional model for two-phase flow. J. Magn. Magn. Mater. 456, 413-422. https://doi.org/10.1016/j.jmmm.2018.02.063 (2018).

19. Ali, F., Imtiaz, A., Khan, I., Sheikh, N. A. \& Ching, D. L. C. Hemodynamic flow in a vertical cylinder with heat transfer: Two-phase Caputo Fabrizio fractional model. J. Magnet. 23(2), 179-191. https://doi.org/10.4283/JMAG.2018.23.2.179 (2018).

20. Ali, F. et al. Two-phase fluctuating flow of dusty viscoelastic fluid between non-conducting rigid plates with heat transfer. IEEE Access 7, 123299-123306. https://doi.org/10.1109/ACCESS.2019.2933529 (2019).

21. Ali, F. et al. A report on fluctuating free convection flow of heat absorbing viscoelastic dusty fluid past in a horizontal channel With MHD effect. Sci. Rep. 10(1), 1-15. https://doi.org/10.1038/s41598-020-65252-1 (2020).

22. Aldoss, T. K. et al. Magnetohydrodynamic mixed convection from a vertical plate embedded in a porous medium. Numer. Heat Transfer Part A Appl. 28(5), 635-645. https://doi.org/10.1080/10407789508913766 (1995).

23. Hossain, A. M. Effect of Hall current on unsteady hydromagnetic free convection flow near an infinite vertical porous plate. J. Phys. Soc. Jpn. 55(7), 2183-2190. https://doi.org/10.1143/JPSJ.55.2183 (1986).

24. Helmy, K. A. MHD unsteady free convection flow past a vertical porous plate. ZAMM J. Appl. Math. Mech./ZeitschriftfürAngewandteMathematik und Mechanik Appl. Math. Mech. 78(4), 255-270. https://doi.org/10.1002/(SICI)1521-4001(199804)78:4\%3c255 $\because$ AID-ZAMM255\%3e3.0.CO;2-V (1998). 
25. Gupta, A. S. Laminar free convection flow of an electrically conducting fluid from a vertical plate with uniform surface heat flux and variable wall temperature in the presence of a magnetic field. ZeitschriftfürangewandteMathematik und Physik ZAMP 13(4), 324-333. https://doi.org/10.1007/BF01601002 (1962).

26. Gupta, A. S. Steady and transient free convection of an electrically conducting fluid from a vertical plate in the presence of a magnetic field. Appl. Sci. Res. 9(1), 319. https://doi.org/10.1007/BF00382210 (1960).

27. Wilks, G. Magnetohydrodynamic free convection about a semi-infinite vertical plate in a strong cross field. ZeitschriftfürangewandteMathematik und Physik ZAMP 27(5), 621-631. https://doi.org/10.1007/BF01591174 (1976).

28. Takhar, H. S., Roy, S. \& Nath, G. Unsteady free convection flow over an infinite vertical porous plate due to the combined effects of thermal and mass diffusion, magnetic field and Hall currents. Heat Mass Transf. 39(10), 825-834. https://doi.org/10.1007/s0023 $1-003-0427-y$ (2003).

29. Ahmed, N., Sarmah, H. K. \& Kalita, D. Thermal diffusion effect on a three-dimensional MHD free convection with mass transfer flow from a porous vertical plate. Latin Am. Appl. Res. 41(2), 165-176 (2011).

30. Sheikh, et al. Comparison and analysis of the Atangana-Baleanu and Caputo-Fabrizio fractional derivatives for generalized Casson fluid model with heat generation and chemical reaction. Results Phys. 7, 789-800. https://doi.org/10.1016/j.rinp.2017.01.025 (2017).

31. Khan, D. et al. Effects of relative magnetic field, chemical reaction, heat generation and Newtonian heating on convection flow of Casson fluid over a moving vertical plate embedded in a porous medium. Sci. Rep. 9(1), 400. https://doi.org/10.1038/s41598-01836243-0 (2019).

32. Sahoo, P. K., Datta, N. \& Biswal, S. Magnetohydrodynamic unsteady free convection flow past an infinite vertical plate with constant suction and heat sink. Indian J. Pure Appl. Math. 34(1), 145-156 (2003).

33. Chamkha, A. J. Unsteady laminar hydromagnetic fluid-particle flow and heat transfer in channels and circular pipes. Int. J. Heat Fluid Flow 21(6), 740-746. https://doi.org/10.1016/S0142-727X(00)00031-X (2000).

34. Kumar, J. P., Umavathi, J. C., Chamkha, A. J. \& Pop, I. Fully-developed free-convective flow of micropolar and viscous fluids in a vertical channel. Appl. Math. Model. 34(5), 1175-1186. https://doi.org/10.1016/j.apm.2009.08.007 (2010).

35. Chamkha, A. J. Hydromagnetic two-phase flow in a channel. Int. J. Eng. Sci. 33(3), 437-446. https://doi.org/10.1016/0020-7225(93) E0006-Q (1995).

36. Chamkha, A. J. Unsteady flow of an electrically conducting dusty-gas in a channel due to an oscillating pressure gradient. Appl. Math. Model. 21(5), 287-292. https://doi.org/10.1016/S0307-904X(97)00018-8 (1997).

37. Chamkha, A. J. On laminar hydromagnetic mixed convection flow in a vertical channel with symmetric and asymmetric wall heating conditions. Int. J. Heat Mass Transf. 45(12), 2509-2525. https://doi.org/10.1016/S0017-9310(01)00342-8 (2002).

38. Gireesha, B. J., Mahesha, S. M. \& Bagewadi, C. S. Hydromagnetic boundary layer flow of rotating dusty fluid under varying pressure gradient. Int. J. Appl. Math. Eng. Sci. 5(2), 123-141 (2011).

39. Manjunatha, S., Gireesha, B. J. \& Bagewadi, C. S. Series solutions for an unsteady flow and heat transfer of a rotating dusty fluid with radiation effect. Acta Math UnivComenianae 86(1), 111-126 (2017).

40. Dey, D. Advances in modelling and analysis A. J. Homepage 55(2), 70-75. http://iieta.org/Journals/AMA/AMAA (2018).

41. Ahmed, N., Goswami, J. K. \& Barua, D. P. MHD transient flow with Hall current past an accelerated horizontal porous plate in a rotating system. Open J. Fluid Dyn. http://www.scirp.org/journal/PaperInformation.aspx?PaperID=40439 (2013).

42. Kanch, A. K. \& Jana, R. N. Hall effects on unsteady hydromagnetic flow past an eccentrically rotating porous disk in a rotating fluid. Czech J. Phys. 49(2), 205-215. https://doi.org/10.1023/A:1022802011874 (1999).

43. Rajagopal, K. R. Flow of viscoelastic fluids between rotating disks. Theoret. Comput. Fluid Dyn. 3(4), 185-206. https://doi. org/10.1007/BF00417912 (1992).

44. Saffman, P. G. On the stability of laminar flow of a dusty gas. J. Fluid Mech. 13(1), 120-128. https://doi.org/10.1017/S002211206 2000555 (1962).

45. Dey, D. Dusty hydromagneticoldryod fluid flow in a horizontal channel with volume fraction and energy dissipation. Int. J. Heat Technol. 34(3), 415-422. https://doi.org/10.18280/ijht.340310 (2016).

46. Dbnath, L. \& Ghosh, A. K. On unsteady hydromagnetic flows of a dusty fluid between two oscillating plates. Appl. Sci. Res. 45(4), 353-365. https://doi.org/10.1007/BF00457067 (1988).

47. Attia, H. A., Al-Kaisy, A. M. A. \& Ewis, K. M. MHD Couette flow and heat transfer of a dusty fluid with exponential decaying pressure gradient. Tamkang J. Sci. Engg. 14(2), 91-96. http://citeseerx.ist.psu.edu/viewdoc/download?doi=10.1.1.464.5682\%26rep $=$ rep $1 \% 26$ type $=$ pdf $(2011)$.

48. Cogley, A. C., Vincent, W. G. \& Gilles, S. E. Differential approximation for radiative transfer in a nongrey gas near equilibrium. AIAA J. 6(3), 551-553 (1968).

49. Cosmstoch, C. The Poincare-Lighthill Perterbation technique and its generalizations. SIAM Rev. 14(3), 433-446. https://doi. org/10.1137/1014069 (1972).

\section{Author contributions}

F.A. model the problem. M.B. and S.K. solved the modeled problem analytically. M.B. and S.K. draw the graphs. Results and discussions have reviewed by M.A. I.K. reviewed the whole manuscript. Proof reading has performed by K.S.N. All authors reviewed the final manuscript.

\section{Competing interests}

The authors declare no competing interests.

\section{Additional information}

Supplementary Information The online version contains supplementary material available at https://doi. org/10.1038/s41598-020-79795-w.

Correspondence and requests for materials should be addressed to F.A.

Reprints and permissions information is available at www.nature.com/reprints.

Publisher's note Springer Nature remains neutral with regard to jurisdictional claims in published maps and institutional affiliations. 
(c) (i) Open Access This article is licensed under a Creative Commons Attribution 4.0 International cc) License, which permits use, sharing, adaptation, distribution and reproduction in any medium or format, as long as you give appropriate credit to the original author(s) and the source, provide a link to the Creative Commons licence, and indicate if changes were made. The images or other third party material in this article are included in the article's Creative Commons licence, unless indicated otherwise in a credit line to the material. If material is not included in the article's Creative Commons licence and your intended use is not permitted by statutory regulation or exceeds the permitted use, you will need to obtain permission directly from the copyright holder. To view a copy of this licence, visit http://creativecommons.org/licenses/by/4.0/.

(C) The Author(s) 2021 